\title{
POR QUE PESQUISAR IMPLEMENTAÇÃO DE POLÍTICAS EDUCACIONAIS ATUALMENTE?
}

José Roberto Rus Perez

\begin{abstract}
RESUMO: Partindo do pressuposto de que ainda continua valendo a distinção das fases da policy (agenda, formulação, implementação e avaliação), este texto apresenta a evolução da reflexão acadêmica e ressalta a relevância dos estudos sobre implementação ainda hoje, assim como traz alguns resultados de pesquisa como uma contribuição para o debate sobre questôes metodológicas na avaliação de processo de implementação, como uma subárea específica da pesquisa de avaliação de política, e para o estudo das políticas educacionais. Serão recuperadas as mudanças e oportunidades do campo da implementação de política educacional como um campo de pesquisa prática, com base na reflexão sobre a literatura produzida e as metodologias empregadas nas pesquisas sobre educação, que se tornou, nos últimos vinte anos, uma arena de acirrados conflitos e interesses com uma política de grandes orçamentos, além de relacionar-se com as múltiplas dimensões do Estado de bem-estar social.
\end{abstract}

Palavras-chave: Política educacional. Implementação. Avaliação de políticas.

\section{EXPLORING THE PRESENT IMPLEMENTATION OF EDUCATIONAL POLICIES: WHAT FOR?}

ABSTRACT: Assuming that it is still valid to distinguish policy into phases (agenda, formulation, implementation and evaluation), this text presents the evolution of the academic reflection

Doutor em Educação e professor livre-docente da Faculdade de Educação da Universidade Estadual de Campinas (UNICAMP).E-mail: rusperez@uol.com.br 
and highlights the relevance of studying implementation today. It also brings forth some research results as a contribution to the debate on methodological issues in the assessment of the implementation process, as a specific subarea of the research on policy assessment for the study of educational policies. Based on a reflection on the literature produced and on the methodology employed in research on education, it retrieves the changes and opportunities in the field of the implementation of educational policy as a field of practical research, which, these last twenty years, has become an arena of fierce conflicts and of interests, because of a large budget policy. It also approaches the multiple dimensions of welfare state.

Key words: Educational policy. Implementation. Policy evaluation.

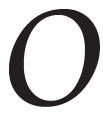

s estudos teóricos sobre metodologias de análise de políticas públicas constituem uma parte importante de minha atividade acadêmica, tanto as de apoio a pesquisas quanto, principalmente, as didáticas.

Procurarei recuperar, aqui, as mudanças e oportunidades do campo da implementação de política educacional como um campo de pesquisa prática, com base na reflexão sobre a literatura produzida e as metodologias por mim empregadas nas pesquisas sobre educação, que se tornou, nos últimos vinte anos, uma arena de acirrados conflitos e interesses com uma política de grandes orçamentos - do governo federal, dos estados e dos municípios - para atender, em tese, todas as crianças e jovens do país, além de relacionar-se com as múltiplas dimensões do Estado de bem-estar social.

Diante dessa complexidade, é importante dedicar-se a examinar a implementação das políticas, assim como reexaminar o que foi implementado e deu certo e o que de fato funciona. Também é importante investigar sob que condiçôes as diversas políticas educacionais são implementadas e como elas funcionam e se são exitosas. Para tanto, têm sido desenvolvidos modelos teoricamente consistentes, visando enfrentar e compreender essa complexidade. Tenho me esforçado para realizar a revisão da literatura sobre implementação, considerada como uma subdisciplina da ciência política e administração pública, e a revisão da literatura específica sobre implementação de políticas educacionais. 
Emergência e consolidação da pesquisa de implementação de políticas educacionais

Parto do pressuposto de que ainda continua valendo a distinção das fases da política (agenda, formulação, implementação e avaliação). Não pretendo, aqui, exaurir o assunto, mas apresentar a evolução da reflexão acadêmica, ressaltar a relevância desse tipo de estudo ainda hoje e, também, trazer alguns resultados de pesquisa como uma contribuição para o debate sobre questóes metodológicas, na avaliação de processo de implementação, como uma subárea específica da pesquisa de avaliação de política, e para o estudo das políticas educacionais.

Para alguns autores, a implementação refere-se a todo o processo iniciado com o estabelecimento da política até o seu impacto; para outros, a implementação não se confunde com o produto, sendo basicamente um processo com uma série de decisóes e de açôes postas pela autoridade legislativa central.

Uma concepção mais delimitada da implementação distingue etapas da policy, pelo menos duas, antecedendo a implementação: a fase da formação da política, implicando a constituição da agenda, a definição do campo de interesse e a identificação de alternativas; e a fase da formulação da política, quando as várias propostas se constituem em política propriamente dita, mediante a definição de metas, objetivos, recursos e a explicitação da estratégia de implementação. Pressman e Wildavsky (1984), em trabalho seminal para a área, delimitam o início do processo de implementação no momento da transformação da política em programa, isto é, quando, por ação de uma autoridade, são criadas as "condiçôes iniciais" à implementação. Porém, esses mesmos autores discutem as dificuldades de se distinguir a implementação da própria política e do programa.

A opção por pesquisar a implementação de políticas educacionais significou não trabalhar com o processo de formulação, que é visto como mais prestigiado do que a implementação, uma vez que a atenção dos analistas se volta mais para a formulação da política do que para os processos operados para as políticas alcançarem os efeitos desejados. Pelo fato de ainda os tomadores de decisão tenderem a assumir que as decisões trazem automaticamente a mudança, conclui-se que a implementação não é vista como uma parte integral da formulação da política, mas como algo a ser feito depois, por acréscimo. Assim, ela 
Por que pesquisar implementação de políticas educacionais atualmente?

não seria valorizada por ser considerada como uma simples sucessão de decisões e interações.

Não se pode deixar de notar que há, ainda, um debate sobre o status da pesquisa de implementação. Há aqueles que não consideram a implementação como um estudo a ser valorizado e outros que entendem que esse tipo de estudo tornou-se fundamental ao longo do tempo. De qualquer forma, a chamada "caixa preta" do processo da política firmou-se como um campo muito fértil de pesquisa.

Como afirmou Peter deLeon, cinquenta anos depois de Lasswell, o termo implementação foi incorporado ao léxico de políticas públicas. Lester and Goggin já tinham concluído que a implementação de política pública continua a sustentar um grande interesse prático para os tomadores de decisão, porque ela é o maior problema ou dificuldade que impede de se atingir os objetivos no processo da política. Assim, muitos analistas políticos consideram que a pesquisa da implementação de política é uma das áreas mais utilizadas da análise de política.

Contudo, Verspoor (1992), ao fazer a revisão de estudos em dezenove países em desenvolvimento, identificou um quase descaso universal pelo tema (issue) da implementação. Também no Brasil, a pesquisa de avaliação era bastante incipiente, sendo que a preocupação com esse tema desenvolveu-se apenas nos anos de 1980 e de forma desigual entre as distintas políticas. Em um balanço das pesquisas nessa área, empreendido por Figueiredo (NEPP, 1989), a autora indicou que, de 144 casos, 6\% avaliaram positivamente os resultados dos programas analisados, nos quais pesaram os fatores institucionais. $25 \%$ dos estudos avaliaram de maneira negativa os programas e a grande maioria situou-se entre estes dois extremos: eram estudos que mostravam tanto os aspectos negativos quanto os positivos para o funcionamento ou efeitos dos programas avaliados. Tomando particularmente a área educacional, como exemplo, a literatura apontava, naquele momento, o quanto as pesquisas acadêmicas estavam distantes da escola, restringindo as avaliações à etapa de diagnóstico e das propostas educacionais, raramente abordando o processo de implementação. Restringiamse ainda as pesquisas aos níveis mais altos da decisão, com pouca vinculação e atenção aos demais níveis da hierarquia administrativa ou das açôes que procuram inovar o ensino. Havia ainda um grande hiato entre o diagnóstico da situação e a tomada de decisão. 
Desde então, a literatura especializada tem chamado a atenção para as contribuições proporcionadas por esse tipo de pesquisa: 1) visam corrigir o curso das ações, fornecendo subsídios aos implementadores durante o curso da política, em razão de suas características de policy oriented; 2) buscam ampliar a efetividade do processo de decisão dos programas públicos; 3) contribuem para ampliar a $a c$ countability dos programas; 4) ampliam a integração dos corpos administrativo, político e comunidade, fundamentais para a mudança do desempenho educacional.

Pode-se dizer que os estudos de políticas tornaram-se ferramentas imprescindíveis para governos elaborarem políticas públicas. A pesquisa de implementação de políticas tem se beneficiado, nos últimos anos, do conjunto de ideias que derivam de quatro décadas de pesquisa, especialmente nos EUA, e constata-se que elas são adequadas a: grande grau de estabilidade política, forte tradição democrática, bases de informação acessíveis para orientar a formulação e burocracia relativamente não corrupta, com forte tradição de avaliação profissional.

A avaliação de políticas, programas e projetos sociais e educacionais foi incorporada, de fato, à agenda governamental brasileira no início dos anos de 1990. Dentre os fatores que contribuíram para isso destacam-se: a consolidação democrática, o ajuste econômico e consequente redução dos recursos para a área social, as maiores exigências impostas pelos órgãos financiadores, especialmente internacionais, em relação ao controle de gastos e resultados etc. Uma dinâmica de racionalização, que incluiu a observância dos critérios de eficácia, efetividade e eficiência na utilização dos recursos financeiros, e uma preocupação crescente com a accountability passaram a envolver a gestão pública brasileira.

Desde o início de meu trabalho no Núcleo de Estudos de Políticas Públicas (NePP), e depois na Faculdade de Educação da UNICAMP, venho aprofundando a discussão a respeito da pesquisa de implementação de programas sociais e, em específico, de políticas educacionais. Nesse momento, a conceituação do processo de implementação ainda gozava de pouco consenso na vasta literatura americana dedicada ao tema. Assim, a expansão das pesquisas de avaliação de política deu-se, principalmente, nos Estados Unidos, a partir dos anos de 1960, no momento de consolidação dos programas de combate à pobreza. Esses programas federais americanos 
Por que pesquisar implementação de políticas educacionais atualmente?

foram um importante marco no desenvolvimento dessas pesquisas, a partir do estudo de caso de Pressman e Wildasky sobre a implementação de um programa na cidade de Oakland (Califórnia), no início daquela década. Entretanto, as bases dessas pesquisas empíricas (inquéritos, avaliação pluridisciplinar), que se relacionaram, primeiramente, aos domínios dos programas educativos e sociais, assentaramse tanto na ação do poder federal do New Deal, quanto na orientação intervencionista das ciências sociais "aplicadas" às consequências da ação governamental.

De fato, desde os anos de 1950, já se desenvolviam pesquisas de avaliação nos Estados Unidos, mediante o uso de enquetes e da análise estatística, numa perspectiva pluridisciplinar, envolvendo as várias ciências sociais, tendo se reforçado essa perspectiva com as políticas sociais governamentais federais dos períodos Kennedy e Jonhson.

As gerações de pesquisas de mplementação que se acumularam demonstram resultados em pelo menos dois aspectos: melhor entendimento do que significa a implementação e sua variação através do tempo, das políticas e das unidades de governo. Houve também avanços realizados no estabelecimento dos elos entre o desempenho da implementação e o planejamento da política.

É interessante verificar o quanto, inicialmente, a ideia de implementação se restringia ao "cumpra-se" da política, uma vez que ela não era considerada no desenho da política, pressupondo que a decisão de uma autoridade seria automaticamente cumprida. $\mathrm{O}$ mérito do estudo de Pressman e Wildavsky foi demonstrar o quanto, apesar da decisão do nível central, a operacionalização do programa apresentava inúmeros percalços no nível local.

Os estudos de implementação também demonstram o quanto esta varia de acordo com a política e seus diferentes tipos de formato, centralizado ou descentralizado, e com a natureza das agências que a implementam. Os estudos indicam a importância de se estabelecer a interrelação dos distintos níveis de governo com as políticas, contrapondo-se à ênfase na decisão de uma única autoridade e numa única política.

Ao efetuarem um balanço das pesquisas, empreendidas entre 1960 e 1985, nos Estados Unidos, Lester e outros (1987) traçaram as características básicas de, pelo menos, três gerações de estudos de 
implementação. A primeira (1970-1975) enfocou detalhadamente os acontecimentos empreendidos por uma única instância de autoridade decisória, caracterizando-se pelos estudos de caso centrados, especialmente, na identificação dos obstáculos à implementação. Os estudos de Jeffrey Pressman e Aaron Wildavsky (1973), Martha Dertick (1972) e Eugene Bardach (1977) dedicaram-se a considerar a imensa quantidade de problemas que existem entre a definição da política e a sua execução. Há uma profusão de estudos de casos, cada um com sua própria lição, mas muito pouco em termos de uma teoria geral da implementação.

A segunda geração de estudos (1975-1980) identificou os sucessos e fracassos da implementação, tendo desenvolvido modelos analíticos, arrolando um conjunto de fatores contribuintes ao sucesso ou ao fracasso dos objetivos da política. Duas abordagens comportaram essa categoria de estudos: a primeira, designada top-down (Van Meter \& Van Horn, 1975; Sebastier \& Maznanian, 1980 e Edwards, 1980), centrada na identificação de um conjunto de variáveis formuladas a partir da decisão política da autoridade governamental central, procurando responder questóes como: o grau de alcance dos objetivos da política; o equacionamento dos impactos e os objetivos; os principais fatores que afetam o impacto e a própria política e as reformulações obtidas ao longo do tempo. A segunda abordagem inclui modelos botton-up (Elmore, 1978; Lipsky, 1971 e 1980), cujos enfoques centraram-se nos atores envolvidos na prestação de serviços locais, tendo sido relevante identificar: os seus objetivos, estratégias, atividades e contatos. Os pressupostos básicos desse modelo são a compatibilidade dos programas aos desejos, vontades e padrões comportamentais dos atores e o processo de descentralização.

Ainda do ponto de vista metodológico, Linder e Peters (1987) consideram que a distinção entre os estudos botton-up e top-down sintetiza as concepções de duas escolas de estudos de implementação. A primeira é basicamente europeia e fenomenológica e a segunda é mais positivista e americana. Embora ambas as abordagens detenham uma concepção instrumental da política, outras diferenças marcam tais estudos, fazendo com que a escola designada top-down evoque uma visão objetiva da política, como produto do desenvolvimento de um processo exógeno, sendo, portanto, objetivamente verificável, contendo "intençôes políticas" que podem ser alteradas e solucionadas. 
Por que pesquisar implementação de políticas educacionais atualmente?

Afora as controvérsias epistemológicas entre o objetivismo e subjetivismo contidas nessas abordagens, Linder e Peters (1987) ainda argumentavam a respeito das consequências desses modelos adotados pelos acadêmicos, no sentido de limitarem o campo de possibilidades que devem ser consideradas pelos formuladores, em outras palavras, as relações entre os estudos acadêmicos e os formuladores de política. Uma estreita concepção de política e dos seus fracassos ou sucesso constrange as opçôes oferecidas pelos acadêmicos aos policy makers, da mesma forma que estes assumem que suas escolhas podem estar determinadas pela suposta probabilidade do sucesso da implementação. Uma concepção mais ampla do sucesso e fracasso, baseada em mais de um critério de desenho da pesquisa, pode incrementar a maneira como a política é considerada, tanto no mundo acadêmico quanto no governamental.

Apesar desse avanço quantitativo e qualitativo, ocorrido principalmente nos EUA, Lester et al. (1987) consideraram que o aprofundamento da pesquisa de avaliação da implementação ainda se defrontava com três obstáculos: pluralismo teórico, contexto restrito e ausência de acúmulo de conhecimento. O pluralismo teórico resultava da inexistência de um modelo que desse conta de explicar adequadamente e de prever o como e o porquê dos resultados das políticas. Para os autores, a pesquisa de implementação era restrita em relação: ao tempo, realizando apenas estudos sincrônicos; à abrangência das análises, realizando apenas estudos de casos isolados; à própria concepção de implementação e sua abordagem teórico-metodológica. Ainda, segundo esses autores, ocorria a ausência de acúmulo de conhecimento, porque cada estudo de caso parecia se encerrar em si mesmo, desconsiderando os demais estudos realizados. Talvez essa forma de crítica esteja demonstrando o caráter bastante localizado e contextualizado desse tipo de pesquisa, característico das microabordagens, por vezes dissociado dos macroprocessos. Assim, é preciso buscar articulações entre os micro e macroprocessos, o que é viável, também, nas pesquisas de implementação.

Paul Sabatier (1986), numa tentativa de síntese dos dois modelos, propõe a análise do processo da política, mas, como observou O'Toole (2000), a controvérsia botom-up/top-down seria apenas um diferente modo de olhar o mesmo fenômeno e que o campo ficou em uma disputa estéril. 
Entre os modelos e esforços para combiná-los, identificam-se quatro paradigmas da implementação de políticas, segundo Matland: implementação administrativa (baixo conflito e baixa ambiguidade); implementação política (alto conflito e baixa ambiguidade); implementação simbólica (alto conflito e alta ambiguidade); e, finalmente, implementação experimental (baixo conflito e alta ambiguidade).

Nos final dos anos 90 do século passado, Peter deLeon constatou que, nos países do Norte, especialmente nos Estados Unidos, vivia-se um renascimento dos estudos de implementação. Nesse período de redescoberta, num artigo provocativo sobre o futuro da pesquisa de implementação, publicado em 1998, James Lester e Malcolm Goggin argumentaram que os estudiosos de implementação podiam ser categorizados em quatro tipos: os "reformers", os "skeptics", os "testers" e os "terminators", dependendo se os estudiosos acreditavam que a pesquisa de implementação deveria continuar ou se tal pesquisa necessitava de modificações substantivas para continuar. Após a publicação do texto, seguiu-se um intenso debate na Policy Currents, da American Political Science Association (APSA), com a participação de inúmeros pesquisadores.

Há ainda uma busca da teoria dedutiva da implementação de política que é testada e que pode ser usada por decision makers para apoiar, suportar suas responsabilidades da implementação. Anne Schneider (1999), respondendo aos dois autores, propõe que a implementação necessita ser estudada como uma variável dependente e como uma variável independente.

Também nesse momento, Michael Hill (1997) questionou se a teoria da implementação seria um tema do passado, para logo dar a essa questão retórica uma resposta negativa.

Com a valorização dos temas (issues) de participação pública, surge a possibilidade de uma abordagem democrática para a implementação da política e que pode se constituir como um foco central do renascimento da implementação, no início do século XXI.

Há aqueles que criticam o fato dos estudos de implementação não incluirem a análise de impacto, não podendo dessa forma determinar o quanto esses fatores (estratégias, práticas de operação etc.) afetam o impacto nos clientes e beneficiários dos programas. Há achados de pesquisas que ilustram a profunda influência que a implementação 
Por que pesquisar implementação de políticas educacionais atualmente?

pode ter na efetividade dos programas sociais; enfim, investir na descoberta do que funciona melhor e para quem.

\section{A pesquisa de implementação de políticas educacionais e suas perspectivas}

A etapa de implementação da política se constituiu num importante objeto de investigação nas pesquisas realizadas pela equipe interdisciplinar de pesquisadores do NEPP, da qual fiz parte, especialmente na extensa investigação sobre "Avaliação do processo de implementaçao do Projeto 'Inovaçôes no Ensino Básico' e de algumas medidas da escola-padrão", que teve por objetivo acompanhar e avaliar, em seus onze subprojetos, o processo de implementação de reformas e inovações educacionais, na rede estadual paulista de ensino na área da Região Metropolitana de São Paulo, ao longo de 24 meses. Na sua primeira fase - julho a dezembro de 1994 -, a investigação examinou o quadro das macropolíticas educacionais, segundo os princípios e a lógica que, até aquele momento, presidiam a política setorial. Na segunda fase janeiro de 1995 a julho de 1996 -, os mesmos programas e medidas foram considerados sob as novas orientações que se lhes foi dada pela gestão da Pasta, iniciada em janeiro de 1995. Além de modificações internas - reconstituídas e analisadas em cada subprojeto que delas trata especificamente -, sua implementação também foi presidida por princípios e diretrizes distintas, impressas na macroestrutura e nas grandes políticas da Secretaria.

Nessa análise do processo de implementação, deu-se ênfase especial à reconstituição da estratégia prevista na introdução da inovação educacional: objetivos que se pretende, consistência na formulação da estratégia, desenho organizacional para viabilizar ações planejadas, busca de apoio e suporte político interno à organização para garantir a implementação. Tratou-se, inicialmente, do escopo e do grau de inovação, ou seja, como foram equacionadas as relações entre estratégia da implementação, estrutura e dinâmica organizacionais. Em seguida, foram identificadas as preferências temporais (no curto, médio e longo prazos) da introdução das reformas e de concretização de seus resultados e a aceitabilidade de riscos quanto ao êxito do processo de reformas, nas opções por maiores conteúdos inovadores ou por mudanças incrementais no sistema. Analisou-se, 
também, a autoridade da reforma, o perfil do grupo reformador e a lógica organizacional para viabilizar as ações planejadas. Finalmente, o modelo concebido tratou de perscrutar a construção de coalizões de apoio, privilegiando atores, sistemas, anéis e mecanismos geradores de apoio e suporte político interno à organização, para garantir a implementação e a existência de supostos sobre o futuro, isto é, a escolha de estratégias frente aos diferentes cenários futuros da introdução das inovações.

A partir dos resultados empíricos dessa pesquisa, foi possível desenvolver pelo menos três dimensões nos modelos de análise do processo de implementação de políticas educacionais, como retratado na literatura especializada. A primeira dimensão trata das relações entre o desenho ou a formulação da política, de um lado, e os formatos que os programas adquirem ao final do processo, de outro. A segunda é a dimensão temporal do processo e seus efeitos diferenciados no tempo sobre a organização em que se processam, sobre os atores que implementam (resistências e adesões) e as modificações das condições iniciais. A terceira refere-se às condições que propiciam ou entravam o processo de implementação.

Ao levar em conta essas dimensões, pode-se esboçar um modelo de pesquisa de processo de implementação que procura captar as relações complexas entre variáveis dependentes (graus e formas da implementação), variáveis intervenientes (comportamento dos agentes envolvidos na implementação) e variáveis independentes (estrutura de operação da rede, envolvendo as dimensões organizacionais, jurídicas, financeiras e de apoio logístico). Além disso, considera-se ainda, nesse nível, a dinâmica de ação dos atores (grau de conhecimento do processo, sistema de incentivo e de punição, opiniōes, interesses etc.). Um modelo dessa natureza pode ter como objetivo o acompanhamento do processo de implementação, identificando os obstáculos e os elementos facilitadores, assim como o grau de adesão e resistência dos agentes implementadores.

Como o processo de implementação dispõe de uma dinâmica no tempo, portanto mutável, é preciso também levar em consideração a comparação entre distintos tempos (inicial, intermediário e final).

Ao que parece, o desenvolvimento dos estudos de processo de implementação aponta, entre nós, para a superação das pesquisas 
Por que pesquisar implementação de políticas educacionais atualmente?

sobre eficácia e desempenho que, basicamente, procuraram cotejar metas, objetivos, com os resultados alcançados pelos programas.

Ao contrário, os modelos mais dinâmicos e processuais pressupõem não apenas a relação da implementação com o contexto mais geral da macropolítica - o que se pode identificar como determinação externa -, como também as relações dinâmicas entre as estruturas organizacionais, os distintos recursos de apoio mobilizados e as características sociais, culturais e econômicas dos atores, que certamente influenciarão o seu comportamento, por serem eles agentes do processo de implementação. Esse conjunto de fatores, interagindo entre si e com a estratégia de implementação, pode afetar o grau de alcance dos resultados e formas da implementação.

Embora os modelos aqui considerados tendam a apoiar-se nos procedimentos experimentais, não se trata de reduzir as possibilidades oferecidas pela pesquisa de avaliação exclusivamente a esses modelos que primam pelo estabelecimento de relação de causalidade, o que é relativamente restrito em se tratando de pesquisa que, certamente, estará lidando com a complexidade de processos políticos e institucionais e com os interesses, opiniōes e graus de adesão e resistência dos atores. É talvez essa complexidade que tem dificultado a discriminação do peso dos distintos "fatores causais" implicados nos modelos abundantemente presentes na literatura americana.

Todos os estudiosos envolvidos com a educação pública reconhecem que a reforma da escola pública urbana pode ser fragmentada e caótica, com propósitos concorrentes e sem um foco claro ou objetivo. Contudo, mesmo quando os objetivos são coerentes, as reformas raramente são implementadas de forma tranquila e total. Há sempre demoras e modificações no processo. Certamente, isso não é privilégio do processo da política educacional. Charles Lindblom observou, décadas atrás, que, mesmo em situação de política coesa, o processo é usualmente realizado por tentativas e erros. Se pensamos o sucesso da política como um contínuo da definição da agenda, para a legislação e para a implementação, os pesquisadores concordam que esse processo quase sempre gera grandes preocupaçóes para as mudanças políticas. Outra conclusão consensual: o problema da implementação é praticamente universal (Goggin et al., 1990; Peters, 1999).

Nessa primeira década do século XXI, a pesquisa de implementação de políticas educacionais aprofundou o conhecimento sobre o 
processo de implementação numa perspectiva epistemológica distinta das pesquisas produzidas no passado, segundo Meredith Honig (2006). O ponto de partida é a consideração de que a política educacional contemporânea difere da política formulada anteriormente, em seu desenho, seus objetivos e metas, sua população-alvo e seus instrumentos.

Se, até meados dos anos 90 do século passado, os pesquisadores identificavam que a análise da implementação da educacional preocupava-se em perguntar por que a política ou programa era implementado ou não, iluminando as variações em três dimensões: políticas, pessoas (implementadores) e lugares; agora, a ênfase está posta na identificação de suas várias dimensões e como e por que a interação dessas três dimensões molda a complexidade do processo de implementação.

Por exemplo, alguns autores têm destacado que a participação das pessoas/implementadores em diversas comunidades e grupos de relacionamentos é essencial para a implementação. Não é outra, senão essa a preocupação de Heather Hill em seu texto Language matters: how characteristics of languages complicate policy implementation (2006), ao mostrar que os professores, no interior de uma escola ou distrito, estão situados em comunidades profissionais que contribuem para, como dirá a autora, moldar suas crenças e visões de mundo e, consequentemente, suas interpretações das mensagens da política.

Recebido em junho de 2010 e aprovado em julho de 2010.

\section{Referências}

BARDACH, E. The implementation game: what happens after a bill becomes a law. 3. ed. Cambridge, Mass.: Cambridge University, 1990.

DELEON, P. The missing link revisited: contemporary implementation research. Policy Studies Review, Urbana, v. 16, n. 3-4, p. 311-318, 1999.

ELMORE, R. Instruments and strategy in public policy. Review of Policy Research, Knoxville, v. 7, n. 1, p. 174-186, 1987. 
Por que pesquisar implementação de políticas educacionais atualmente?

GOGGIN, M. et al. Implementation theory and practice: towards a third generation. Glenview: Foresman, Little \& Brown, 1990.

HILL, H.C. Language matters: how characteristics of language complicate policy implementation. In: Honing, M.I. New directions in education policy implementation: confronting complexity. New York: SUNY, 2006. p. 65-82.

HILL, M. Implementation theory: yesterday's issue? Policy and Politics, Bristol, v. 25, n. 4, p. 375-385, 1997.

HONING, M.I. New directions in education policy implementation: confronting complexity. New York: sUNY, 2006.

LESTER, J.P. et al. Public policy implementation: evolution of the field and agenda for future research. Policy Studies Review, Urbana, v. 7, n. 1, p. 200-216, 1987.

LESTER, J.P.; GOGGIN, M. Back to the future: the rediscovery of implementation studies. Policy Currents, Albuquerque, v. 8, n. 3, p. $1-9,1998$.

LINDBLON, C. O processo de decisão política. Brasília, DF: UNB, 1981.

LINDER, S.H.; PETERS, B.G. Relativism, contingency and the definition of success in implementation research. Policy Studies Review, Urbana, v. 7, n. 1, p. 116-127, 1987.

MATLAND, R. Sinthetesizing the implementation literature: the ambiguity-conflict model of policy implementation. Journal of Public Administration Research and Theory, Lawrence, v. 5, n. 2, p. 145-174, 1995.

NÚCLEO DE ESTUDOS DE POLÍTICAS PÚBLICAS (NEPP). $A$ política social no estado de São Paulo: bibliografia comentada. Campinas: NEPP/UNICAMP, 1989.

NÚCLEO DE ESTUDOS DE POLÍTICAS PÚBLICAS (NEPP). Avaliação do processo de implementação do Projeto "Inovações no Ensino Básico" e de algumas medidas da escola-padrão. Campinas: UNICAMP, 1995. Relatório final de pesquisa. (mimeo). 
O'TOOLE JUNIOR, L.J. Research on policy implementation: assessment and prospects. Journal of Public Administration Research and Theory, Lawrence, v. 10, n. 2, p. 263-88, 2000.

PRESSMAN, J.; WILDAVSKY, A. Implementation. 3. ed. Berkeley: University of California, 1984.

RUS PEREZ, J.R. Avaliação do processo de implementação: algumas questões metodológicas. In: Rıco, E.M. (Org.). Avaliação de políticas sociais: uma questão em debate. São Paulo: Cortez; Instituto de Estudos Especiais, 1998a. p. 65-73.

RUS PEREZ, J.R. Reflexões sobre a avaliação do processo de implementação de políticas e programas educacionais. In: WARDE, M.J. (Org.). Novas políticas educacionais: perspectivas e críticas. São Paulo: PUC-SP, 1998b. v. 1. p. 139-145.

SABATIER, P.A. Top-down and botton-up approaches to implementation research: a critical analysis and suggested synthesis. Journal of Public Policy, Cambridge, v. 6, n. 1, p. 21-48, 1986.

SABATIER, P.A.; MAZMANIAN, D.A. Policy implementation: a framework of analysis. Policy Studies Journal, Urbana, v. 8, p. 538560, 1980 .

SCHNEIDER, A.L. Terminator! Who, me?: some thoughts about the study of policy implementation. Policy Currents, Albuquerque, v. 9, n. 1, p. 1-5, 1999 . 